\title{
MAXIMUM LIKELIHOOD ESTIMATOR OF THE VOLATILITY OF FORWARD RATES DRIVEN BY GEOMETRIC SPATIAL AR SHEET
}

\author{
JÓZSEF GÁLL, GYULA PAP, AND MARTIEN C. A. VAN ZUIJLEN
}

Received 29 June 2003 and in revised form 23 March 2004

Discrete-time forward interest rate curve models are studied, where the curves are driven by a random field. Under the assumption of no-arbitrage, the maximum likelihood estimator of the volatility parameter is given and its asymptotic behaviour is studied. First, the so-called martingale models are examined, but we will also deal with the general case, where we include the market price of risk in the discount factor.

\section{Introduction}

In this paper, we study estimation problems in interest rate and bond pricing structures. In the literature, one can find several approaches to the formulation of interest rate structures and based on them, one can derive prices of bonds and other interest-ratedependent financial assets. An overview on this subject is given, for example, in [12].

The models we consider are based on an idea of Heath et al. [8]. They constructed a continuous-time model for the so-called forward rate structures and derived the bond prices from this structure. Later on, many authors studied such forward rate-based bond models. In what follows, such models will be referred to as Heath-Jarrow-Morton (HJM)type models. We note that in the literature, the HJM-type models differ in the parametrization. We follow the so-called Musiela parametrisation (see, e.g., [11] or [1]), in which the basic model can be summarized as follows.

Let $f(t, x)$ denote the instantaneous forward rate at time $t$ with time to maturity $x$, where $x, t \in \mathbb{R}_{+}$, where $\mathbb{R}_{+}$denotes the set of the nonnegative real numbers. In particular, the spot interest rate is defined by $r(t):=f(t, 0), t \in \mathbb{R}_{+}$. In this HJM-type model, the forward rates are assumed to follow the dynamics

$$
d f(t, x)=\alpha(t, x) d t+\sigma(t, x) d W(t),
$$

where $\{W(t)\}_{t \in \mathbb{R}_{+}}$is a standard Wiener process. In an integral form, we have

$$
f(t, x)=f(0, x)+\int_{0}^{t} \alpha(u, x) d u+\int_{0}^{t} \sigma(u, x) d W(u) .
$$

Copyright (C) 2004 Hindawi Publishing Corporation Journal of Applied Mathematics 2004:4 (2004) 293-309 2000 Mathematics Subject Classification: 91B28, 62F12, 62F10

URL: http://dx.doi.org/10.1155/S1110757X04306133 
We emphasise again that we follow the Musiela parametrisation, and hence $x$ is time to maturity and not time of maturity. Having built up the forward rate dynamics, the common way in the literature to define the price of a zero-coupon bond at time $t$ with maturity date $s$ is to take

$$
P(t, s):=\exp \left\{-\int_{0}^{s-t} f(t, u) d u\right\}, \quad 0 \leq t \leq s .
$$

One can see in the above model that for any value $x \geq 0$ in (1.1), the forward rate process $\{f(t, x)\}_{t \in \mathbb{R}_{+}}$is driven by the same Wiener process. To put it in another way, one can say that the same "shocks" have effect on all the forward rates, which seems not to be very realistic. Therefore, it is natural to generalise the classical models by introducing a random driving field instead of a single driving process. In this way, forward rates with different times to maturity can be driven by different processes.

Such a generalisation of the classical HJM-type models has been proposed by Kennedy [10] in the continuous case. Later on, several authors studied such random field models; here we refer to Goldstein [6] and Santa-Clara and Sornette [14]. We can formulate the main idea of random field models as follows. Let $\{Z(t, s)\}_{t, s \in \mathbb{R}_{+}}$be a random field and suppose that for each fixed $x \in \mathbb{R}_{+}$, the forward rate dynamics is given by

$$
d f(t, x)=\alpha(t, x) d t+\sigma(t, x) Z(d t, x)
$$

where $\{Z(t, s)\}_{t \in \mathbb{R}_{+}}$is a martingale for any $s \geq 0$. Writing (1.4) in an integral form, we have

$$
f(t, x)=f(0, x)+\int_{0}^{t} \alpha(u, x) d u+\int_{0}^{t} \sigma(u, x) Z(d u, x) .
$$

In contrast to a "random field" model like (1.4), a model of the form (1.1) will be called "classical."

A major part of defining such a model is to find appropriate driving processes or driving fields for the forward rates. Although in the classical models, Brownian motions are the most commonly used driving processes (see, e.g., [8]), more general models are also known in the literature. Schmidt [15] proposed for instance a natural generalisation of the Brownian motion, namely, the Ornstein-Uhlenbeck process, which can be considered as the natural analogue of an autoregressive $(A R)(1)$ process in discrete time. Sometimes, some further considerations can be taken into account-especially in the random field case - which help us to find appropriate and more realistic candidates. Typically, the covariance structure of the driving field can be restricted by further assumptions, as described, for example, in $[6,14]$. Knowing the classical models, it is not surprising to see that Brownian sheets and also integrated Brownian sheets and Ornstein-Uhlenbeck sheets are quite usually used in the random field case. See Kennedy [10], Goldstein [6], or Santa-Clara and Sornette [14]. Note that in [14], some further examples are also studied.

The HJM model (see [8]) as well as the models studied in $[6,10,14]$ are continuoustime models. One can find several papers on the discrete versions of the classical HJM 
models. Here we mention $[7,9,13]$. Like in the classical case, it is reasonable and sensible to model and investigate possible discrete-time counterparts of the continuous-time random field models of the form (1.4). In [4], such discrete-time random field models have been studied.

In this paper-based on the models and results of [4] —-we consider discrete-time random forward interest rate models, where the forward rates corresponding to different times to maturity are driven by a Gaussian type of random field, which has been built up by a system of i.i.d. Gaussian random variables. Keeping in mind the consideration on the possible continuous-time random fields mentioned above, we will study models which are equipped with the natural discrete-time analogues of these fields, that is, we will study a Gaussian field built up in an autoregressive way (Section 2). With the special choice of this driving process, one can get back the classical models as well as a simple discrete spatial Gaussian lattice, which could be the most natural analogue of many continuous-time random field models. To make the models realistic, one has to claim that the market excludes arbitrage opportunities. In [4], such models have been proposed and also no-arbitrage conditions have been derived for these models. Therefore, in this paper, we will always assume that the interest rate curves satisfy the no-arbitrage conditions. First, we will focus in our study on the so-called "martingale" case (see Sections 3 and 4), where the market measure is an equivalent measure. Such an approach appears in derivative pricing problems in the literature, among others in $[2,3]$. In our case, this assumption implies a drift condition (see [4]).

In this setting, our aim is to find an appropriate estimator for the volatility parameter of the model and to study its asymptotic behaviour. Assuming that the volatility parameters are deterministic and independent of time and of maturity and also that the i.i.d. random variables involved are standardly normally distributed, we will find the maximum likelihood estimator of the volatility parameter (Section 3) together with its asymptotic distribution (Section 4). Depending on the value of the autoregression parameter, we will separate the stable and unstable (or nearly unit root) case and obtain results for both cases.

Furthermore, in contrast to the martingale case, we will study, say, a "general" case in Section 5. For this, a more complicated model must be used, in which market price of risk will be introduced as a new factor. Again, based on the no-arbitrage conditions, we will see that the technique applied in Sections 3 and 4 can be used to derive similar results as in the "martingale" case.

We also mention another important source of motivation for studying discrete-time forward rates driven by random fields. It is the problem that the rigorous definitions of some notions of the continuous counterpart models have not been worked out yet in the literature because of certain technical or theoretical difficulties caused by the change from the classical models to random field structures. Discrete approximation provides a promising way for solving these problems. Some results on this question are given by the authors [5], where it is discussed that Ornstein-Uhlenbeck sheets can be obtained as a limit of the discrete-time autoregression models, which are studied in this paper. So this is another reason why we focus on autoregression models in this paper. 


\section{The model and the no-arbitrage criterion}

We will treat discrete-time forward interest rate curve models driven by a spatial autoregressive process. The model can be built up as follows. Let $\left\{\eta(i, j): i, j \in \mathbb{Z}_{+}\right\}$be a system of i.i.d. random variables with mean zero and variance one on a probability space $(\Omega, \mathscr{F}, \mathbb{P})$. Introduce the filtration $\mathscr{F}_{k}:=\sigma\left(\eta(i, j): 0 \leq i \leq k, j \in \mathbb{Z}_{+}\right), k \in \mathbb{Z}_{+}$. Consider the doubly geometric spatial autoregressive process $\left\{S(k, \ell): k, \ell \in \mathbb{Z}_{+}\right\}$generated by

$$
\begin{gathered}
S(k, \ell)=S(k-1, \ell)+\varrho S(k, \ell-1)-\varrho S(k-1, \ell-1)+\eta(k, \ell), \\
S(k,-1)=S(-1, \ell)=0
\end{gathered}
$$

where $\varrho \in \mathbb{R}$. Then a discrete-time forward interest rate curve model with initial values $\left\{f(0, \ell): \ell \in \mathbb{Z}_{+}\right\}$, with coefficients $\left\{\alpha(k, \ell), \beta(k, \ell): k, \ell \in \mathbb{Z}_{+}\right\}$, and with driving process $\left\{S(k, \ell): k, \ell \in \mathbb{Z}_{+}\right\}$is given by

$$
f(k+1, \ell)=f(0, \ell)+\sum_{i=0}^{k} \alpha(i, \ell)+\sum_{i=0}^{k} \beta(i, \ell) \Delta_{1} S(i, \ell), \quad k, \ell \in \mathbb{Z}_{+},
$$

where $\Delta_{1} S(i, \ell):=S(i+1, \ell)-S(i, \ell)$, the random variables $\left\{\alpha(k, \ell), \beta(k, \ell): \ell \in \mathbb{Z}_{+}\right\}$are $\mathscr{F}_{k}$-measurable, and $f(0, \ell) \in \mathbb{R}, \ell \in \mathbb{Z}_{+}$. Clearly $\left\{f(k, \ell): k, \ell \in \mathbb{Z}_{+}\right\}$satisfies the (stochastic) difference equation

$$
f(k+1, \ell)=f(k, \ell)+\alpha(k, \ell)+\beta(k, \ell) \Delta_{1} S(k, \ell), \quad k, \ell \in \mathbb{Z}_{+}
$$

The random variable $f(k, \ell)$ is in fact the instantaneous forward rate at time $k$ with time to maturity $\ell$. Hence, the (spot) interest rate holding for the time period $t=k$ to $t=k+1$ is defined by

$$
r(k):=f(k, 0) \quad \forall k \in \mathbb{Z}_{+}
$$

Clearly

$$
S(k, \ell)=\sum_{i=0}^{k} \sum_{j=0}^{\ell} \varrho^{\ell-j} \eta(i, j)
$$

and hence

$$
\Delta_{1} S(k, \ell)=\sum_{j=0}^{\ell} \varrho^{\ell-j} \eta(k+1, j)
$$

Using this equation, one can easily check that

$$
\begin{aligned}
\operatorname{cov}\left(\Delta_{1} S\left(k, \ell_{1}\right), \Delta_{1} S\left(k, \ell_{2}\right)\right) & =\sum_{j_{1}=0}^{\ell_{1}} \sum_{j_{2}=0}^{\ell_{2}} \varrho^{\ell_{1}+\ell_{2}-j_{1}-j_{2}} \operatorname{cov}\left(\eta\left(k+1, j_{1}\right), \eta\left(k+1, j_{2}\right)\right) \\
& =\sum_{j=0}^{\ell_{1} \wedge \ell_{2}} \varrho^{\ell_{1}+\ell_{2}-2 j}= \begin{cases}\frac{\varrho^{\ell_{1}+\ell_{2}+2}-\varrho^{\left|\ell_{1}-\ell_{2}\right|}}{\varrho^{2}-1} & \text { for } \varrho \neq \pm 1, \\
\left(\left(\ell_{1} \wedge \ell_{2}\right)+1\right) \varrho^{\ell_{1}+\ell_{2}} & \text { for } \varrho= \pm 1 .\end{cases}
\end{aligned}
$$


Consequently, the covariances

$$
\operatorname{cov}\left(\Delta_{1} S\left(k, \ell_{1}\right), \Delta_{1} S\left(k, \ell_{2}\right)\right)=: c\left(\ell_{1}, \ell_{2}\right)
$$

do not depend on $k$.

For the sake of simplicity, we suppose that the stochastic discount factor process $\left\{M(k): k \in \mathbb{Z}_{+}\right\}$is given by $M(0):=1$ and

$$
M(k+1)=e^{-f(k, 0)} M(k), \quad k \in \mathbb{Z}_{+} .
$$

The price $P(k, \ell)$ of a zero-coupon bond at time $k \in \mathbb{Z}_{+}$with maturity $\ell \in \mathbb{Z}_{+}$with $\ell \geq k$ is defined by $P(k, k):=1$ and

$$
P(k, \ell+1)=e^{-f(k, \ell-k)} P(k, \ell), \quad k, \ell \in \mathbb{Z}_{+}, k \leq \ell .
$$

This is a discrete-time analogue of formula (1.3), defined now in a recursive way.

As is natural in financial mathematics, we are interested only in models where arbitrage opportunities are excluded in the market. The no-arbitrage conditions are based on the existence of an equivalent martingale measures. In this paper, we will study volatility estimation in the "martingale" case, where the real measure of the market is assumed to be a martingale measure. A similar approach has been proposed and studied by Föllmer and Sondermann [3], and Föllmer and Schweizer [2]. In this martingale case, a drift condition occurs which makes the volatility estimation complicated. Note that several no-arbitrage criteria for the model at issue have been derived by the authors [4]. For our martingale case and under the assumption that the common distribution of $\left\{\eta(i, j): i, j \in \mathbb{Z}_{+}\right\}$is the standard normal distribution, it is proved [4, Corollary 2, page 14] that the no-arbitrage criterion implies

$$
f(k, \ell+1)=f(k, 0)+\sum_{j=0}^{\ell} \alpha(k, j)-\frac{1}{2} \sum_{j_{1}=0}^{\ell} \sum_{j_{2}=0}^{\ell} \beta\left(k, j_{1}\right) \beta\left(k, j_{2}\right) c\left(j_{1}, j_{2}\right)
$$

for all $k, \ell \in \mathbb{Z}_{+}$. From (2.11), one can obtain the difference equation

$$
f(k, \ell+1)=f(k, \ell)+\alpha(k, \ell)-\frac{1}{2} \beta(k, \ell)^{2} c(\ell, \ell)-\beta(k, \ell) \sum_{j=0}^{\ell-1} \beta(k, j) c(\ell, j) .
$$

Together with (2.2), we obtain

$$
\begin{aligned}
f(k+1, \ell)-f(k, \ell+1)= & \frac{1}{2} \beta(k, \ell)^{2} c(\ell, \ell)+\beta(k, \ell) \sum_{j=0}^{\ell-1} \beta(k, j) c(\ell, j) \\
& +\beta(k, \ell) \Delta_{1} S(k, \ell) .
\end{aligned}
$$


298 MLE of volatility of forward rates driven by AR sheet

\section{ML estimation in martingale models}

We consider a forward interest rate curve model $\left\{f(k, \ell): k, \ell \in \mathbb{Z}_{+}\right\}$given in (2.2). Suppose that the common distribution of $\left\{\eta(i, j): i, j \in \mathbb{Z}_{+}\right\}$is the standard normal distribution and the model satisfies the no-arbitrage criterion (2.11). Assume that there exists $\beta \in \mathbb{R}, \beta \neq 0$, such that $\beta(k, \ell)=\beta$ a.s. for all $k, \ell \in \mathbb{Z}_{+}$.

In the lemma below, we will obtain, based on the forward rates, an explicit expression for the maximum likelihood estimator of the volatility.

Lemma 3.1. Assume that the parameter $\varrho$ is known. Let $K$ and $L$ be positive integers. Then the maximum likelihood estimator ${\widehat{\beta^{2}}}_{K, L}$ of $\beta^{2}$ based on the sample

$$
\{f(k, \ell): 1 \leq k \leq K, 0 \leq \ell \leq L\}
$$

is given by

$$
{\widehat{\beta^{2}}}_{K, L}:=\frac{-B_{K, L}+\sqrt{B_{K, L}^{2}+4 A_{K, L} C_{K, L}}}{2 A_{K, L}}
$$

where

$$
\begin{aligned}
A_{K, L}: & =\frac{K}{4} \sum_{\ell=0}^{L-1}\left(\sum_{i=0}^{2 \ell} \varrho^{i}\right)^{2}+\frac{1}{4} \sum_{k=1}^{K} \frac{1}{k}\left(\sum_{j=0}^{k-1} \sum_{i=0}^{2 L+2 j} \varrho^{i}\right)^{2}, \\
B_{K, L}: & =K(L+1), \\
C_{K, L}: & =\sum_{k=1}^{K} \sum_{\ell=0}^{L-1} g_{k, \ell}^{2}+\sum_{k=1}^{K} \frac{1}{k} \tilde{g}_{k, L}^{2},
\end{aligned}
$$

where

$$
\begin{aligned}
& g_{k, \ell}:= \begin{cases}f(k, \ell)-f(k-1, \ell+1)-\varrho(f(k, \ell-1)-f(k-1, \ell)) & \text { for } k, \ell \geq 1, \\
f(k, 0)-f(k-1,1) & \text { for } k \geq 1, \ell=0,\end{cases} \\
& \tilde{g}_{k, L}:=f(k, L)-f(0, k+L)-\varrho(f(k, L-1)-f(0, k+L-1)) \quad \text { for } k, L \geq 1 \text {. }
\end{aligned}
$$

Proof. The aim of the following discussion is to find the joint density of $\{f(k, \ell): 1 \leq$ $k \leq K, 0 \leq \ell \leq L\}$. By (2.13), we have

$$
f(k+1, \ell)-f(k, \ell+1)=\frac{1}{2} \beta^{2} c(\ell, \ell)+\beta^{2} \sum_{j=0}^{\ell-1} c(\ell, j)+\beta \Delta_{1} S(k, \ell), \quad k, \ell \in \mathbb{Z}_{+} .
$$

Clearly

$$
\sum_{j=0}^{\ell-1} c(\ell, j)=\sum_{j=0}^{\ell-1} \sum_{i=0}^{j} \varrho^{\ell+j-2 i}
$$


hence

$$
c(\ell, \ell)+2 \sum_{j=0}^{\ell-1} c(\ell, j)=\left(\sum_{i=0}^{\ell} \varrho^{i}\right)^{2}
$$

Using (2.6), we observe

$$
f(k+1, \ell)-f(k, \ell+1)=\frac{\beta^{2}}{2}\left(\sum_{i=0}^{\ell} \varrho^{i}\right)^{2}+\beta \sum_{j=0}^{\ell} \varrho^{\ell-j} \eta(k+1, j),
$$

hence

$$
f(k+1, \ell)-f(k, \ell+1)-\varrho(f(k+1, \ell-1)-f(k, \ell))=\frac{\beta^{2}}{2} \sum_{i=0}^{2 \ell} \varrho^{i}+\beta \eta(k+1, \ell)
$$

for $k \geq 0, \ell \geq 1$. Consequently, $f(k+1, \ell)$ can be expressed by $f(k, \ell+1), f(k+1, \ell-1)$, $f(k, \ell)$, and $\eta(k+1, \ell)$, and the conditional distribution of $f(k+1, \ell)$, given $f(k, \ell+1)$, $f(k+1, \ell-1)$, and $f(k, \ell)$, is a normal distribution with mean

$$
f(k, \ell+1)+\varrho(f(k+1, \ell-1)-f(k, \ell))+\frac{\beta^{2}}{2} \sum_{i=0}^{2 \ell} \varrho^{i}
$$

and variance $\beta^{2}$. Moreover,

$$
f(k+1,0)-f(k, 1)=\frac{\beta^{2}}{2}+\beta \eta(k+1,0), \quad k \geq 0,
$$

hence the conditional distribution of $f(k+1,0)$, given $f(k, 1)$, is a normal distribution with mean $f(k, 1)+\beta^{2} / 2$ and variance $\beta^{2}$. Finally,

$$
f(k+1, \ell)=f(0, k+\ell+1)+\frac{\beta^{2}}{2} \sum_{j=0}^{k}\left(\sum_{i=0}^{\ell+j} \varrho^{i}\right)^{2}+\beta \sum_{j=0}^{k} \sum_{i=0}^{k+\ell-j} \varrho^{k+\ell-j-i} \eta(j+1, i),
$$

which implies

$$
\begin{gathered}
f(k+1, \ell)-f(0, k+\ell+1)-\varrho(f(k+1, \ell-1)-f(0, k+\ell)) \\
=\frac{\beta^{2}}{2} \sum_{j=0}^{k} \sum_{i=0}^{2 \ell+2 j} \varrho^{i}+\beta \sum_{j=0}^{k} \eta(j+1, k+\ell-j) .
\end{gathered}
$$

Consequently, $f(k+1, \ell)$ can be expressed by $f(0, k+\ell+1), f(k+1, \ell-1), f(0, k+\ell)$, and $\{\eta(j+1, k+\ell-j): 0 \leq j \leq k\}$, and the conditional distribution of $f(k+1, \ell)$, given $f(0, k+\ell+1), f(k+1, \ell-1)$, and $f(0, k+\ell)$, is a normal distribution with mean

$$
f(0, k+\ell+1)+\varrho(f(k+1, \ell-1)-f(0, k+\ell))-\frac{\beta^{2}}{2} \sum_{j=0}^{k} \sum_{i=0}^{2 \ell+2 j} \varrho^{i}
$$


and variance $(k+1) \beta^{2}$. For $\{f(k, \ell): 1 \leq k \leq K, 1 \leq \ell \leq L-1\}$ we use the first conditional distribution, for $\{f(k, 0): 1 \leq k \leq K\}$ the second one, and for $\{f(k, L): 1 \leq k \leq K\}$ we use the third one. By the independence of $\left\{\eta(i, j): i, j \in \mathbb{Z}_{+}\right\}$, we obtain that the joint density $h\left(x_{k, \ell}: 1 \leq k \leq K, 0 \leq \ell \leq L\right)$ of $\{f(k, \ell): 1 \leq k \leq K, 0 \leq \ell \leq L\}$ has the form

$$
\begin{aligned}
\frac{1}{\left(2 \pi \beta^{2}\right)^{(L+1) K / 2}(K !)^{1 / 2}} \exp \{ & -\frac{1}{2 \beta^{2}} \sum_{k=1}^{K} \sum_{\ell=0}^{L-1}\left(y_{k, \ell}-\frac{\beta^{2}}{2} \sum_{i=0}^{2 \ell} \varrho^{i}\right)^{2} \\
& \left.-\frac{1}{2 \beta^{2}} \sum_{k=1}^{K} \frac{1}{k}\left(\tilde{y}_{k, L}-\frac{\beta^{2}}{2} \sum_{j=0}^{k-1} \sum_{i=0}^{2 L+2 j} \varrho^{i}\right)^{2}\right\},
\end{aligned}
$$

where $y_{k, \ell}$ and $\tilde{y}_{k, L}$ are defined by

$$
\begin{aligned}
& y_{k, \ell}:= \begin{cases}x_{k, \ell}-x_{k-1, \ell+1}-\varrho\left(x_{k, \ell-1}-x_{k-1, \ell}\right) & \text { for } k, \ell \geq 1, \\
x_{k, 0}-x_{k-1,1} & \text { for } k \geq 1, \ell=0,\end{cases} \\
& \tilde{y}_{k, L}:=x_{k, L}-x_{0, k+L}-\varrho\left(x_{k, L-1}-x_{0, k+L-1}\right) \quad \text { for } k, L \geq 1 \text {, }
\end{aligned}
$$

and $x_{0, \ell}:=f(0, \ell)$ for $\ell \geq 0$.

Thus the maximum likelihood estimator ${\widehat{\beta^{2}}}_{K, L}$ of $\beta^{2}$ can be obtained by minimizing

$$
K(L+1) \log \beta^{2}+\frac{1}{\beta^{2}} \sum_{k=1}^{K} \sum_{\ell=0}^{L-1}\left(y_{k, \ell}-\frac{\beta^{2}}{2} \sum_{i=0}^{2 \ell} \varrho^{i}\right)^{2}+\frac{1}{\beta^{2}} \sum_{k=1}^{K} \frac{1}{k}\left(\tilde{y}_{k, L}-\frac{\beta^{2}}{2} \sum_{j=0}^{k-1} \sum_{i=0}^{2 L+2 j} \varrho^{i}\right)^{2} .
$$

Taking the derivative with respect to $\beta^{2}$, we obtain

$$
\begin{aligned}
& \frac{K(L+1)}{\beta^{2}}-\frac{1}{\beta^{4}} \sum_{k=1}^{K} \sum_{\ell=0}^{L-1}\left(y_{k, \ell}-\frac{\beta^{2}}{2} \sum_{i=0}^{2 \ell} \varrho^{i}\right)^{2}-\frac{1}{\beta^{2}} \sum_{k=1}^{K} \sum_{\ell=0}^{L-1}\left(y_{k, \ell}-\frac{\beta^{2}}{2} \sum_{i=0}^{2 \ell} \varrho^{i}\right) \sum_{i=0}^{2 \ell} \varrho^{i} \\
& -\frac{1}{\beta^{4}} \sum_{k=1}^{K} \frac{1}{k}\left(\tilde{y}_{k, L}-\frac{\beta^{2}}{2} \sum_{j=0}^{k-1} \sum_{i=0}^{2 L+2 j} \varrho^{i}\right)^{2}-\frac{1}{\beta^{2}} \sum_{k=1}^{K} \frac{1}{k}\left(\tilde{y}_{k, L}-\frac{\beta^{2}}{2} \sum_{j=0}^{k-1} \sum_{i=0}^{2 L+2 j} \varrho^{i}\right) \sum_{j=0}^{k-1} \sum_{i=0}^{2 \ell+2 j} \varrho^{i} \\
& =\frac{K(L+1)}{\beta^{2}}-\frac{1}{\beta^{4}} \sum_{k=1}^{K} \sum_{\ell=0}^{L-1}\left(y_{k, \ell}-\frac{\beta^{2}}{2} \sum_{i=0}^{2 \ell} \varrho^{i}\right)\left(y_{k, \ell}+\frac{\beta^{2}}{2} \sum_{i=0}^{2 \ell} \varrho^{i}\right) \\
& -\frac{1}{\beta^{4}} \sum_{k=1}^{K} \frac{1}{k}\left(\tilde{y}_{k, L}-\frac{\beta^{2}}{2} \sum_{j=0}^{k-1} \sum_{i=0}^{2 L+2 j} \varrho^{i}\right)\left(\tilde{y}_{k, L}+\frac{\beta^{2}}{2} \sum_{j=0}^{k-1} \sum_{i=0}^{2 L+2 j} \varrho^{i}\right) \\
& =\frac{K(L+1)}{\beta^{2}}-\frac{1}{\beta^{4}} \sum_{k=1}^{K} \sum_{\ell=0}^{L-1}\left(y_{k, \ell}^{2}-\frac{\beta^{4}}{4}\left(\sum_{i=0}^{2 \ell} \varrho^{i}\right)^{2}\right)-\frac{1}{\beta^{4}} \sum_{k=1}^{K} \frac{1}{k}\left(\tilde{y}_{k, L}^{2}-\frac{\beta^{4}}{4}\left(\sum_{j=0}^{k-1} \sum_{i=0}^{2 L+2 j} \varrho^{i}\right)^{2}\right) .
\end{aligned}
$$


Consequently, $\widehat{\beta^{2}}{ }_{K, L}$ is a solution of the equation

$$
A_{K, L} \beta^{4}+B_{K, L} \beta^{2}-C_{K, L}=0
$$

This is a second-order equation for ${\widehat{\beta^{2}}}_{K, L}$, and its positive root gives the maximum likelihood estimator of $\beta^{2}$.

\section{Asymptotic behaviour of the volatility estimator}

Consider a sequence of discrete-time forward interest rate curve models $\left\{f_{n}(k, \ell): k, \ell \in\right.$ $\left.\mathbb{Z}_{+}\right\}, n \in \mathbb{N}$, with initial values $\left\{f_{n}(0, \ell): \ell \in \mathbb{Z}_{+}\right\}$, with coefficients $\left\{\alpha_{n}(k, \ell), \beta_{n}(k, \ell)\right.$ : $\left.k, \ell \in \mathbb{Z}_{+}\right\}$, and with driving process $\left\{S_{n}(k, \ell): k, \ell \in \mathbb{Z}_{+}\right\}$with parameter $\varrho_{n}$. Assume that there exists $\beta_{n} \in \mathbb{R}, \beta_{n} \neq 0$, such that $\beta_{n}(k, \ell)=\beta_{n}$ a.s. for all $k, \ell \in \mathbb{Z}_{+}$. Suppose that the common distribution of $\left\{\eta_{n}(i, j): i, j \in \mathbb{Z}_{+}\right\}, n \in \mathbb{N}$, is the standard normal distribution for each model $\left\{f_{n}(k, \ell): k, \ell \in \mathbb{Z}_{+}\right\}, n \in \mathbb{N}$, and the no-arbitrage condition (2.11) is satisfied in the models.

We will study two important cases regarding the behaviour of the autoregression parameter $\varrho_{n}$. First, we consider a so-called nearly unit root (or unstable) case where the autoregression parameter $\varrho_{n}$ tends to 1 . Secondly, we study the stable case, where the sequence $\varrho_{n}(n \in \mathbb{N})$ has a limit $\varrho$ with $|\varrho|<1$. Theorem 4.1 summarises our main result achieved in the unstable case.

THeORem 4.1. Consider the maximum likelihood estimator ${\widehat{\beta^{2}}}_{K_{n}, L_{n}}$ of $\beta_{n}^{2}$ based on a sample $\left\{f_{n}(k, \ell): 1 \leq k \leq K_{n}, 0 \leq \ell \leq L_{n}\right\}$, where $K_{n}=n K+o(n)$ and $L_{n}=n L+o(n)$ as $n \rightarrow \infty$ with some $K, L>0$. Assume that $\varrho_{n}=1+\gamma / n+o\left(n^{-1}\right)$ as $n \rightarrow \infty$, where $\gamma \in \mathbb{R}$, and $\liminf _{n \in \mathbb{N}}\left|\beta_{n}\right|>0$. Then

$$
n^{2} \beta_{n}^{-1}\left({\widehat{\beta^{2}}}_{K_{n}, L_{n}}-\beta_{n}^{2}\right) \stackrel{\mathscr{D}}{\longrightarrow} \mathcal{N}\left(0,4 \sigma^{2}\right)
$$

where

$$
\frac{1}{\sigma^{2}}:=K \int_{0}^{L}\left(\int_{0}^{2 t} e^{\gamma v} d v\right)^{2} d t+\int_{0}^{K} \frac{1}{s}\left(\int_{0}^{s} \int_{0}^{2 L+2 u} e^{\gamma v} d v d u\right)^{2} d s
$$

Proof. We have

$$
{\widehat{\beta^{2}}}_{K, L}-\beta^{2}=\frac{2\left(C_{K, L}-\beta^{4} A_{K, L}-\beta^{2} B_{K, L}\right)}{B_{K, L}+2 \beta^{2} A_{K, L}+\sqrt{B_{K, L}^{2}+4 A_{K, L} C_{K, L}}} .
$$

Clearly, we also have

$$
\begin{aligned}
& A_{K_{n}, L_{n}}=\frac{n^{4}}{4}\left(\frac{1}{\sigma^{2}}+o(1)\right), \\
& B_{K_{n}, L_{n}}=n^{2}(K L+o(1)),
\end{aligned}
$$


as $n \rightarrow \infty$. Moreover,

$$
\begin{aligned}
C_{K, L}= & \sum_{k=0}^{K-1}(f(k+1,0)-f(k, 1))^{2} \\
& +\sum_{k=0}^{K-1} \sum_{\ell=1}^{L-1}(f(k+1, \ell)-f(k, \ell+1)-\varrho(f(k+1, \ell-1)-f(k, \ell)))^{2} \\
& +\sum_{k=0}^{K-1} \frac{1}{k+1}(f(k+1, L)-f(0, k+L+1)-\varrho(f(k+1, L-1)-f(0, k+L)))^{2} .
\end{aligned}
$$

Applying (3.9), (3.11), and (3.12), we obtain

$$
\begin{aligned}
C_{K, L}-\beta^{4} A_{K, L}= & \beta^{2} \sum_{k=0}^{K-1} \sum_{\ell=0}^{L-1} \eta(k+1, \ell)^{2}+\beta^{2} \sum_{k=0}^{K-1} \frac{1}{k+1}\left(\sum_{j=0}^{k} \eta(j+1, k+L-j)\right)^{2} \\
& +\beta^{3} \sum_{k=0}^{K-1} \sum_{\ell=0}^{L-1} \eta(k+1, \ell) \sum_{i=0}^{2 \ell} \varrho^{i}+\beta^{3} \sum_{k=0}^{K-1} \frac{1}{k+1} \sum_{j=0}^{k} \eta(j+1, k+L-j) \sum_{j=0}^{k} \sum_{i=0}^{2 L+2 j} \varrho^{i} .
\end{aligned}
$$

Dividing by $n^{2}$, the first two terms converge in probability to some deterministic limit since

$$
\begin{gathered}
\frac{1}{n^{2}} \sum_{k=0}^{K_{n}-1} \sum_{\ell=0}^{L_{n}-1} \eta_{n}(k+1, \ell)^{2} \stackrel{L_{1}}{\longrightarrow} K L, \\
\frac{1}{n^{2}} \sum_{k=0}^{K_{n}-1} \frac{1}{k+1}\left(\sum_{j=0}^{k} \eta_{n}(j+1, k+\ell-j)\right)^{2} \stackrel{L_{1}}{\longrightarrow} 0 .
\end{gathered}
$$

Dividing by $n^{2}$, the third and fourth terms have a limit in distribution, namely,

$$
\begin{aligned}
& \frac{1}{n^{2}} \sum_{k=0}^{K_{n}-1} \sum_{\ell=0}^{L_{n}-1} \eta_{n}(k+1, \ell) \sum_{i=0}^{2 \ell} \varrho_{n}^{i} \stackrel{\mathscr{D}}{=} \mathcal{N}\left(0, \frac{1}{n^{4}} \sum_{k=0}^{K_{n}-1} \sum_{\ell=0}^{L_{n}-1}\left(\sum_{i=0}^{2 \ell} \varrho_{n}^{i}\right)^{2}\right) \stackrel{\mathscr{D}}{\longrightarrow} \mathcal{N}\left(0, K \int_{0}^{L}\left(\int_{0}^{2 t} e^{\gamma v} d v\right)^{2} d t\right), \\
& \frac{1}{n^{2}} \sum_{k=0}^{K_{n}-1} \frac{1}{k+1} \sum_{j=0}^{k} \eta_{n}\left(j+1, k+L_{n}-j\right) \sum_{j=0}^{k} \sum_{i=0}^{2 L_{n}+2 j} \varrho_{n}^{i} \\
& \stackrel{\mathscr{D}}{=} \mathcal{N}\left(0, \frac{1}{n^{4}} \sum_{k=0}^{K_{n}-1} \frac{1}{k+1}\left(\sum_{j=0}^{k} \sum_{i=0}^{2 L_{n}+2 j} \varrho_{n}^{i}\right)^{2}\right) \\
& \stackrel{\mathscr{D}}{\longrightarrow} \mathcal{N}\left(0, \int_{0}^{K} \frac{1}{s}\left(\int_{0}^{s} \int_{0}^{2 L+2 u} e^{\gamma v} d v d u\right)^{2} d s\right) .
\end{aligned}
$$


Independence of the third and fourth terms implies

$$
\frac{1}{\beta_{n}^{3} n^{2}}\left(C_{K_{n}, L_{n}}-\beta_{n}^{4} A_{K_{n}, L_{n}}-\beta_{n}^{2} B_{K_{n}, L_{n}}\right) \stackrel{\mathscr{D}}{\longrightarrow} \mathcal{N}\left(0, \frac{1}{\sigma^{2}}\right)
$$

since limsup $\operatorname{su\mathbb {N}}_{n \in \mathbb{N}} 1 /\left|\beta_{n}\right|<\infty$. Furthermore,

$$
\frac{1}{\beta_{n}^{2} n^{4}}\left(B_{K_{n}, L_{n}}+2 \beta_{n}^{2} A_{K_{n}, L_{n}}\right) \longrightarrow \frac{1}{2 \sigma_{K, L}^{2}} \quad \text { as } n \longrightarrow \infty .
$$

Finally,

$$
\begin{aligned}
B_{K_{n}, L_{n}}^{2}+4 A_{K_{n}, L_{n}} C_{K_{n}, L_{n}}= & \left(B_{K_{n}, L_{n}}+2 \beta_{n}^{2} A_{K_{n}, L_{n}}\right)^{2} \\
& +4 A_{K_{n}, L_{n}}\left(C_{K_{n}, L_{n}}-\beta_{n}^{4} A_{K_{n}, L_{n}}-\beta_{n}^{2} B_{K_{n}, L_{n}}\right),
\end{aligned}
$$

hence

$$
\frac{1}{\beta_{n}^{2} n^{4}} \sqrt{B_{K_{n}, L_{n}}^{2}+4 A_{K_{n}, L_{n}} C_{K_{n}, L_{n}}} \stackrel{\mathbb{P}}{\longrightarrow} \frac{1}{2 \sigma_{K, L}^{2}} .
$$

By (4.10), (4.11), and (4.13), we obtain the statement.

Remark 4.2. If $\beta_{n} \rightarrow \beta$ with $\beta \neq 0$, then

$$
n^{2}\left({\widehat{\beta^{2}}}_{K_{n}, L_{n}}-\beta_{n}^{2}\right) \stackrel{\mathscr{D}}{\longrightarrow} \mathcal{N}\left(0,4 \beta^{2} \sigma^{2}\right) .
$$

Moreover, for $\gamma=0$, we have

$$
\frac{1}{\sigma^{2}}=\frac{K}{12}\left(4 L^{3}+4 L K^{2}+K^{3}\right),
$$

and for $\gamma \neq 0$,

$$
\frac{1}{\sigma^{2}}=\frac{K}{\gamma^{2}} \int_{0}^{L}\left(e^{2 \gamma t}-1\right)^{2} d t+\frac{1}{\gamma^{2}} \int_{0}^{K} \frac{1}{s}\left(\int_{0}^{s}\left(e^{2 \gamma(L+u)}-1\right) d u\right)^{2} d s .
$$

The following statements can be useful to derive asymptotic interval estimation for the volatility.

COROLlary 4.3. Under the assumption of Theorem 4.1,

$$
n^{2}{\widehat{\beta^{2}}}_{K_{n}, L_{n}}^{-1 / 2}\left({\widehat{\beta^{2}}}_{K_{n}, L_{n}}-\beta_{n}^{2}\right) \stackrel{\mathscr{D}}{\longrightarrow} \mathcal{N}\left(0,4 \sigma^{2}\right) .
$$

Proof. To show this, first note that

$$
\frac{\beta_{n}^{2}}{\widehat{\beta^{2}}} \stackrel{\mathbb{P}}{\longrightarrow} 1
$$

Indeed, from Theorem 4.1 , one can easily obtain that $\beta_{n}^{-2}\left({\widehat{\beta^{2}}}_{K_{n}, L_{n}}-\beta_{n}^{2}\right) \stackrel{\mathbb{P}}{\rightarrow} 0$. Now, (4.18) and (4.1) together with Slutsky's lemma lead us to the desired statement. 
Next, we turn to the study of the stable case. Our main result regarding the stable case is presented in the following theorem.

THEOREM 4.4. Consider the maximum likelihood estimator ${\widehat{\beta^{2}}}_{K_{n}, L_{n}}$ of $\beta_{n}^{2}$ based on a sample $\left\{f_{n}(k, \ell): 1 \leq k \leq K_{n}, 0 \leq \ell \leq L_{n}\right\}$, where $K_{n}=n K+o(n)$ and $L_{n}=n L+o(n)$ as $n \rightarrow \infty$ with some $K, L>0$. Assume that $\varrho_{n} \rightarrow \varrho$, where $\varrho \in(-1,1)$ and $\beta_{n} \rightarrow \beta \in \mathbb{R}$ as $n \rightarrow \infty$. Then

$$
n\left({\widehat{\beta^{2}}}_{K_{n}, L_{n}}-\beta_{n}^{2}\right) \stackrel{\mathscr{D}}{\longrightarrow} \mathcal{N}\left(0, \frac{2 \beta^{4}}{2 \beta^{2} \lambda+K L}\right),
$$

where

$$
\lambda=\frac{K(2 L+K)}{8(1-\varrho)^{2}} .
$$

Proof. To obtain the desired result, as in the proof of Theorem 4.1, we will study the asymptotics of the terms appearing in (4.3). First, note that

$$
\begin{aligned}
A_{K_{n}, L_{n}}= & \frac{K_{n}}{4\left(1-\varrho_{n}\right)^{2}}\left(L_{n}-2 \varrho_{n} \frac{1-\varrho_{n}^{2 L_{n}}}{1-\varrho_{n}^{2}}+\varrho_{n}^{2} \frac{1-\varrho_{n}^{4 L_{n}}}{1-\varrho_{n}^{4}}\right) \\
& +\frac{1}{4\left(1-\varrho_{n}\right)^{2}} \sum_{k=1}^{K_{n}} \frac{1}{k}\left(k-\varrho_{n}^{2 L_{n}+1} \frac{1-\varrho_{n}^{2 k}}{1-\varrho_{n}^{2}}\right)^{2} \\
= & \frac{K(2 L+K)}{8(1-\varrho)^{2}} n^{2}+o\left(n^{2}\right)
\end{aligned}
$$

and $B_{K_{n}, L_{n}}=K L n^{2}+o\left(n^{2}\right)$ as $n \rightarrow \infty$. Next, define for $0 \leq k<K_{n}, 0 \leq \ell \leq L_{n}, n \in \mathbb{N}$,

$$
\xi_{n}(k, \ell):=\left\{\begin{aligned}
\beta_{n}^{3} \eta_{n}(k+1, \ell) \sum_{i=0}^{2 \ell} \varrho_{n}^{i}+\beta_{n}^{2}\left(\eta_{n}(k+1, \ell)^{2}-1\right) & \text { if } 0 \leq \ell<L_{n}, \\
\beta_{n}^{3} \frac{1}{\sqrt{k+1}} \sum_{j=0}^{k} \eta_{n}(j+1, k+L-j) \frac{1}{\sqrt{k+1}} \sum_{j=0}^{k} \sum_{i=0}^{2 L_{n}+2 j} \varrho_{n}^{i} & \\
+\beta_{n}^{2}\left(\left(\frac{1}{\sqrt{k+1}} \sum_{j=0}^{k} \eta_{n}(j+1, k+L-j)\right)^{2}-1\right) & \text { if } \ell=L_{n} .
\end{aligned}\right.
$$

Recalling (4.7), one can write

$$
C_{K_{n}, L_{n}}-\beta_{n}^{4} A_{K_{n}, L_{n}}-\beta_{n}^{2} B_{K_{n}, L_{n}}=\sum_{k=0}^{K_{n}-1} \sum_{\ell=0}^{L_{n}} \xi_{n}(k, \ell) .
$$

It is easy to see that

$$
\begin{aligned}
& \frac{1}{n^{2}} \operatorname{Var}\left(C_{K_{n}, L_{n}}-\right.\left.\beta_{n}^{4} A_{K_{n}, L_{n}}-\beta_{n}^{2} B_{K_{n}, L_{n}}\right) \longrightarrow \frac{K(2 L+K) \beta^{6}}{2(1-\varrho)^{2}}+2 K L \beta^{4}, \\
& \frac{1}{n^{4}} \sum_{k=0}^{K_{n}-1} \sum_{\ell=0}^{L_{n}} \mathbb{E} \xi_{n}(k, \ell)^{4} \longrightarrow 0
\end{aligned}
$$


as $n \rightarrow \infty$. Hence, by Lyapounov's limit theorem, we obtain from (4.24) that

$$
\frac{1}{n}\left(C_{K_{n}, L_{n}}-\beta_{n}^{4} A_{K_{n}, L_{n}}-\beta_{n}^{2} B_{K_{n}, L_{n}}\right) \stackrel{\mathscr{D}}{\longrightarrow} \mathcal{N}\left(0, \frac{K(2 L+K) \beta^{6}}{2(1-\varrho)^{2}}+2 K L \beta^{4}\right) .
$$

Furthermore,

$$
\begin{gathered}
\frac{1}{n^{2}}\left(B_{K_{n}, L_{n}}+2 \beta_{n}^{2} A_{K_{n}, L_{n}}\right) \longrightarrow K L+\frac{K(2 L+K) \beta^{2}}{4(1-\varrho)^{2}}, \\
\frac{1}{n^{2}} \sqrt{B_{K_{n}, L_{n}}^{2}+4 A_{K_{n}, L_{n}} C_{K_{n}, L_{n}}} \stackrel{\mathbb{P}}{\longrightarrow} K L+\frac{K(2 L+K) \beta^{2}}{4(1-\varrho)^{2}},
\end{gathered}
$$

as $n \rightarrow \infty$. By combining (4.25), (4.26), we obtain the statement.

In order to derive asymptotic interval estimation for the volatility in the stable case, one can apply the following corollary.

Corollary 4.5. Under the assumption of Theorem 4.4, suppose that $\beta \neq 0$. Then

$$
n\left(\frac{2{\widehat{\beta^{2}}}_{K_{n}, L_{n}} \lambda+K L}{2\left({\widehat{\beta^{2}}}_{K_{n}, L_{n}}\right)^{2}}\right)^{1 / 2}\left({\widehat{\beta^{2}}}_{K_{n}, L_{n}}-\beta_{n}^{2}\right) \stackrel{\mathscr{D}}{\longrightarrow} \mathcal{N}(0,1)
$$

with $\lambda$ given by (4.20).

Proof. It can be obtained as an easy consequence of (4.19) that $\widehat{\beta^{2}}{ }_{K_{n}, L_{n}} \stackrel{\mathbb{P}}{\rightarrow} \beta^{2}$, thus Slutsky's lemma and (4.19) lead to (4.27).

\section{A general case}

As we mentioned in the introduction, our main focus in the previous sections was on studying the so-called martingale case. In this section, we turn to the consideration of the general case, where the asset price processes discounted by the corresponding interest rates are no longer supposed to be martingales. Instead, we introduce a new factor, called "market price of risk," which modifies (and generalises) the discount factor. The model we will study in this section is the one introduced in [4], which we summarise next.

For this, recall the autoregressive field defined in (2.1) and take $\phi_{j} \in \mathbb{R}$ for $j \in \mathbb{Z}_{+}$ such that $\sum_{j=0}^{\infty} \phi_{j} \Delta_{1} S(k, j)$ is convergent with probability one. Note that, for example, $\sum_{j=0}^{\infty} \phi_{j}^{2}<\infty$ and $|\varrho|<1$ would be sufficient for this convergence, but one can certainly find other sufficient conditions. Now, consider a general discount factor satisfying

$$
M(k+1)=M(k) \exp \left\{-r(k)+\sum_{j=0}^{\infty} \phi_{j} \Delta_{1} S(k, j)\right\}, \quad k \in \mathbb{Z}_{+},
$$

where the factors $\phi_{j}$ 's will be called market prices of risk. As usual, one should claim the market to exclude arbitrage. Hence, the bond price processes discounted by the above discount factors are claimed to form martingales. No-arbitrage conditions for the model 
at issue have been derived in [4]. The authors found that in case of forward rates defined by (2.1) and (2.2) and equipped with the discount factor in (5.1), the no-arbitrage implies

$$
\begin{aligned}
f(k, \ell+1)= & f(k, \ell)+\alpha(k, \ell)-\frac{1}{2} \beta(k, \ell)^{2} c(\ell, \ell)-\beta(k, \ell) \sum_{j=0}^{\ell-1} \beta(k, j) c(\ell, j) \\
& +\beta(k, \ell) \sum_{j=0}^{\infty} \phi_{j} c(\ell, j), \quad k, \ell \in \mathbb{Z}_{+},
\end{aligned}
$$

where $c(\ell, j), \ell, j \in \mathbb{Z}_{+}$, are defined in (2.7) and (2.8). Note that (5.2) is the generalisation of (2.12) in the martingale case.

For more on such models and the role of the market price of risk, we refer to $[1,4,14]$. We also note that Santa-Clara and Sornette derived the continuous counterpart of (5.2) (see [14]). Furthermore, the interested reader can find results on the limiting connection of such discrete and continuous models in [5].

Since the driving fields follow an autoregressive structure, which implies a "geometric" feature (see, e.g., (2.6)), we will suppose that the market price of risk parameters behave in a similar way. Therefore, in what follows, we assume that

$$
\phi_{j}=\beta b q^{j}, \quad j \in \mathbb{Z}_{+},
$$

where $b \in \mathbb{R}$ and $|q|<1$ such that $|q \varrho|<1$. Note that the latter condition is sufficient for the convergence of $\sum_{j=0}^{\infty} \phi_{j} \Delta_{1} S(k, j)$ with probability one. The parameter $b$ is included for the sake of generality, although the assumption $b=1$ would already lead to a quite general model. The reason why $\phi_{j}$ is defined relative to $\beta$ will be discussed later on.

Now we turn to the maximum likelihood estimator of the volatility.

Lemma 5.1. Consider a forward interest rate curve model $\left\{f(k, \ell): k, \ell \in \mathbb{Z}_{+}\right\}$given in (2.2) and suppose that (5.1) together with the no-arbitrage conditions (5.2) and (5.3) are valid. Assume that $\varrho, b, q$ are known. Then under the assumptions of Lemma 3.1 taken on the parameters and the sample, the maximum likelihood estimator ${\widehat{\beta^{2}}}_{K, L}$ of $\beta^{2}$ is given by

$$
{\widehat{\beta^{2}}}_{K, L}:=\frac{-B_{K, L}+\sqrt{B_{K, L}^{2}+4 A_{K, L} C_{K, L}}}{2 A_{K, L}},
$$

where

$$
\begin{aligned}
A_{K, L}:= & \frac{K}{4} \sum_{\ell=0}^{L-1}\left[\sum_{i=0}^{2 \ell} \varrho^{i}-2 b \frac{q^{\ell}}{1-q \varrho}\right]^{2} \\
& +\frac{1}{4} \sum_{k=1}^{K} \frac{1}{k}\left[\sum_{j=0}^{k-1} \sum_{i=0}^{2 L+2 j} \varrho^{i}-2 b \frac{q^{L}\left(1-q^{k}\right)}{(1-q \varrho)(1-q)}\right]^{2}
\end{aligned}
$$

and $B_{K, L}, C_{K, L}$ are the same as in Lemma 3.1. 
Proof. One can derive the statement of this lemma by following the steps of the proof of Lemma 3.1, where one should use (5.2) instead of (2.12) of course. Hence we omit here the details of the proof.

The market price of risk is defined relative to the volatility in our setup. One could of course parametrise the market price of risk without the inclusion of the volatility. However, we remark that with the inclusion of the volatility, the maximum likelihood estimator will not be a solution of a second-order equation and cannot be expressed explicitly. On the other hand, it is important to emphasise that this way does not cause any loss of generality. It is, in fact, just a matter of parametrisation.

Next, we should like to examine the asymptotics of the estimator given in Lemma 5.1. For this, like in Section 4, consider again a sequence of discrete-time forward interest rate curve models $\left\{f_{n}(k, \ell): k, \ell \in \mathbb{Z}_{+}\right\}, n \in \mathbb{N}$, (with parameters $\beta_{n}, \varrho_{n}, b_{n}, q_{n}$ ) which fulfill (5.2) with discount factors (5.1).

Theorem 5.2. Suppose that $b=\lim _{n \rightarrow \infty} b_{n}$ with $b \in \mathbb{R}$, and $q=\lim _{n \rightarrow \infty} q_{n}$ with $\left|q_{n}\right|<1$, $\left|q_{n} \varrho_{n}\right|<1$ for all $n \in \mathbb{N}$. Furthermore, assume that the driving process $S_{n}$ and the sample size parameters $K_{n}, L_{n}$ are as in Theorem 4.1 .

(a) If $|q|<1$ and the parameters $\beta_{n}, \varrho_{n}$ are as in Theorem 4.1, then statement (4.1) remains valid with $\sigma^{2}$ given by (4.2).

(b) If $|q|<1$ and the parameters $\beta_{n}, \varrho_{n}$ are as in Theorem 4.4, then statement (4.19) remains valid with $\lambda$ given by (4.20).

(c) If $q_{n}=1-\kappa / n+o\left(n^{-1}\right)$, with some $\kappa \in \mathbb{R}$ (and hence $q=1$ ), and the parameters $\beta_{n}$, $\varrho_{n}$ are as in Theorem 4.1 with $\kappa>\gamma$, then statement (4.1) is valid with

$$
\begin{aligned}
\frac{1}{\sigma^{2}}:= & K \int_{0}^{L}\left(\int_{0}^{2 t} e^{\gamma v} d v-\frac{2 b e^{-\kappa t}}{\kappa-\gamma}\right)^{2} d t \\
& +\int_{0}^{K} \frac{1}{s}\left(\int_{0}^{s} \int_{0}^{2 L+2 u} e^{\gamma v} d v d u-\frac{2 b e^{-\kappa L}}{\kappa-\gamma} \int_{0}^{s} e^{-\kappa v} d v\right)^{2} d s
\end{aligned}
$$

(d) If $q_{n}=1-\kappa / n+o\left(n^{-1}\right)$, with some $\kappa \in \mathbb{R}$ (and hence $\left.q=1\right)$, and the parameters $\beta_{n}$, $\varrho_{n}$ are as in Theorem 4.4, then statement (4.19) is valid with

$$
\begin{aligned}
\lambda= & \frac{K}{4(1-\varrho)^{2}}\left(L-2 b \int_{0}^{L} e^{-\kappa t} d t\right)^{2} \\
& +\frac{1}{4(1-\varrho)^{2}} \int_{0}^{K} \frac{1}{s}\left(s-2 b e^{-\kappa L} \int_{0}^{s} e^{-\kappa t} d t\right)^{2} d s
\end{aligned}
$$

Proof. For the proof, one can follow and repeat the steps of the proofs of Theorems 4.1 and 4.4. The only part we should like to emphasise is the asymptotic behaviour of $A_{K_{n}, L_{n}}$. In case (a), the limit of $n^{-4} A_{K_{n}, L_{n}}$ remains the limit given in (4.4) despite the fact that $A_{K_{n}, L_{n}}$ is now given by Lemma 5.1. Similarly, the limit of $n^{-2} A_{K_{n}, L_{n}}$ in case (b) remains the limit given in (4.21). In case (c) and case (d), however, we obtain different limits for $n^{-4} A_{K_{n}, L_{n}}$ and $n^{-2} A_{K_{n}, L_{n}}$, respectively, which leads to the normal limit distributions given above. 
We note finally that one could, of course, take some other forms for the market price of risk. Even much simpler models than (5.3) could be examined. For example, finitely many factors $\phi_{0}, \ldots, \phi_{N} \neq 0$ could be considered (with $\phi_{j}=0$ for $j>N$ ) or even a singlefactor case, that is, $\phi_{0} \neq 0, \phi_{j}=0, j>0$, could be of interest. In this sense, the classical (not random field-based) models are all such single-factor models. In the literature, authors suggest that the market price of risk parameter(s) could be observed in the market possibly by the aid of other financial assets (since they should be considered as some common feature of the market). For a general discussion on the role of the market price of risk, one can consult, for example, [1].

\section{Acknowledgments}

This research has been supported by the Hungarian Scientific Research Fund under Grant no. OTKA-T032361/2000. The authors thank the fund for this support. The authors are also thankful to the unknown referee whose useful remarks and suggestions have been used for the improvement of this paper.

\section{References}

[1] T. Björk, Arbitrage Theory in Continuous Time, Oxford University Press, Oxford, 1998.

[2] H. Föllmer and M. Schweizer, Hedging of contingent claims under incomplete information, Applied Stochastic Analysis (London, 1989) (M. H. A. Davis and R. J. Elliott, eds.), Stochastics Monogr., vol. 5, Gordon and Breach, New York, 1991, pp. 389-414.

[3] H. Föllmer and D. Sondermann, Hedging of nonredundant contingent claims, Contributions to Mathematical Economics (W. Hildenbrand and A. Mas-Colell, eds.), North-Holland, Amsterdam, 1986, pp. 205-223.

[4] J. Gáll, G. Pap, and M. C. A. van Zuijlen, Forward interest rate curves in discrete time settings driven by random fields, Tech. Report 0213, University of Nijmegen, Nijmegen, 2002.

[5] L Limiting connection between discrete and continuous time forward interest rate curve models, Acta Appl. Math. 78 (2003), no. 1-3, 137-144.

[6] R. S. Goldstein, The term structure of interest rates as a random field, Review of Financial Studies 13 (2000), no. 2, 365-384.

[7] D. Heath, R. A. Jarrow, and A. Morton, Bond pricing and the term structure of interest rates: a discrete time approximation, Journal of Financial and Quantitative Analysis 25 (1990), no. 4, 419-440.

[8] _ Bond pricing and the term structure of interest rates: a new methodology for contingent claims valuation, Econometrica 60 (1992), no. 1, 77-105.

[9] R. A. Jarrow, Modelling Fixed Income Securities and Interest Rate Options, McGraw-Hill, New York, 1996.

[10] D. P. Kennedy, The term structure of interest rates as a Gaussian random field, Math. Finance 4 (1994), no. 3, 247-258.

[11] M. Musiela and M. Rutkowski, Martingale Methods in Financial Modelling, Applications of Mathematics, vol. 36, Springer-Verlag, Berlin, 1997.

[12] A. Pelsser, Efficient Methods for Valuing Interest Rate Derivatives, Springer Finance, SpringerVerlag, London, 2000.

[13] S. R. Pliska, Introduction to Mathematical Finance: Discrete Time Models, Blackwell Publishers, Massachusetts, 1997.

[14] P. Santa-Clara and D. Sornette, The dynamics of the forward interest rate curve with stochastic string shocks, Review of Financial Studies 14 (2001), no. 1, 149-185. 
[15] W. M. Schmidt, On a general class of one-factor models for the term structure of interest rates, Finance Stoch. 1 (1997), no. 1, 3-24.

József Gáll: Faculty of Informatics, University of Debrecen, P.O. Box 12, 4010 Debrecen, Hungary E-mail address: jgall@inf.unideb.hu

Gyula Pap: Faculty of Informatics, University of Debrecen, P.O. Box 12, 4010 Debrecen, Hungary E-mail address: papgy@inf.unideb.hu

Martien C. A. van Zuijlen: Department of Mathematics, University of Nijmegen, Toernooiveld 1, 6525 ED Nijmegen, The Netherlands

E-mail address: zuijlen@math.kun.nl 


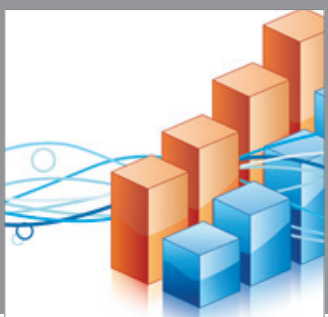

Advances in

Operations Research

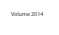

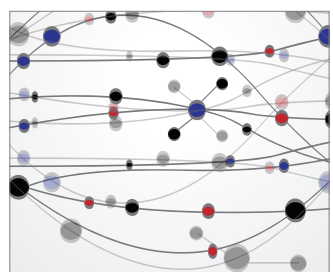

\section{The Scientific} World Journal
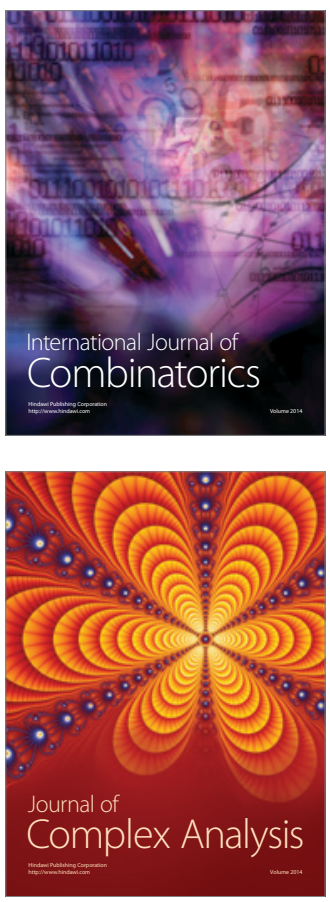

International Journal of

Mathematics and

Mathematical

Sciences
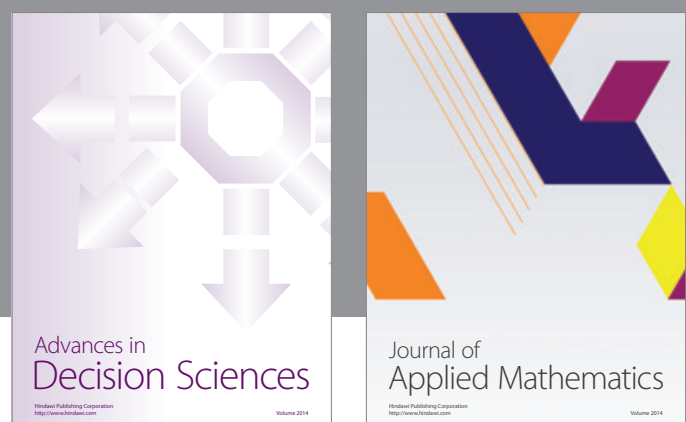

Journal of

Applied Mathematics
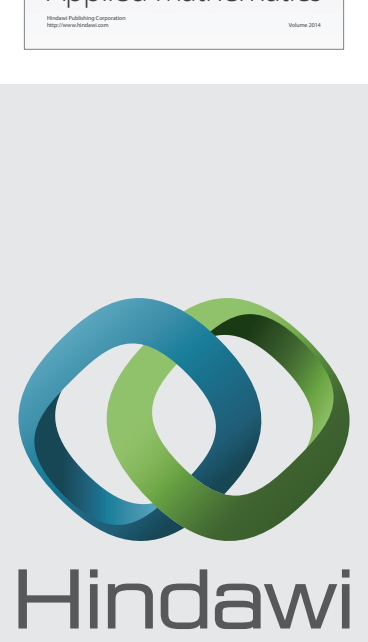

Submit your manuscripts at http://www.hindawi.com
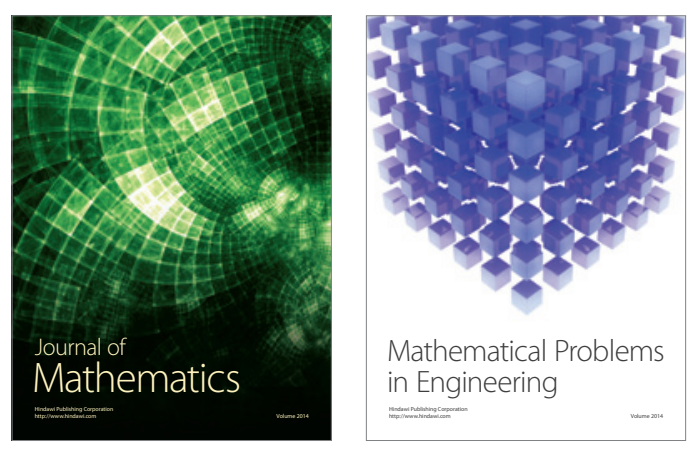

Mathematical Problems in Engineering
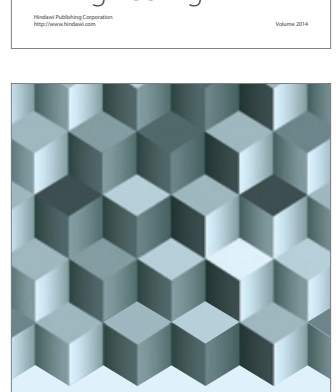

Journal of

Function Spaces
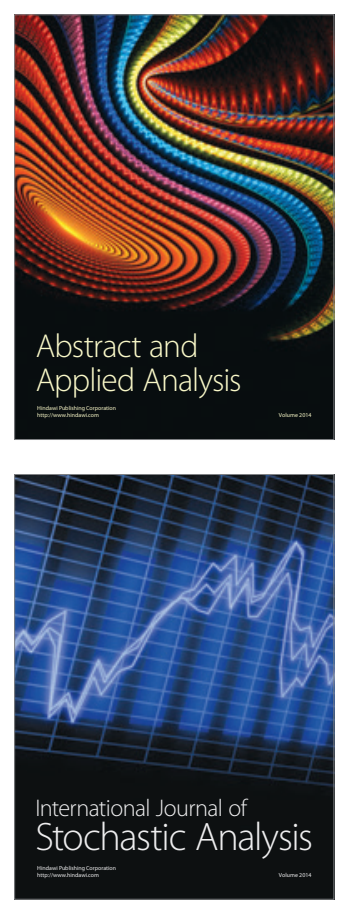

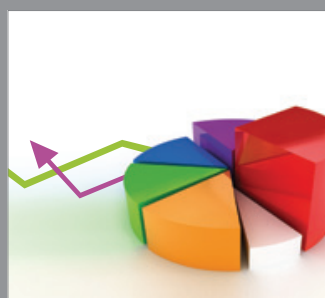

ournal of

Probability and Statistics

Promensencen
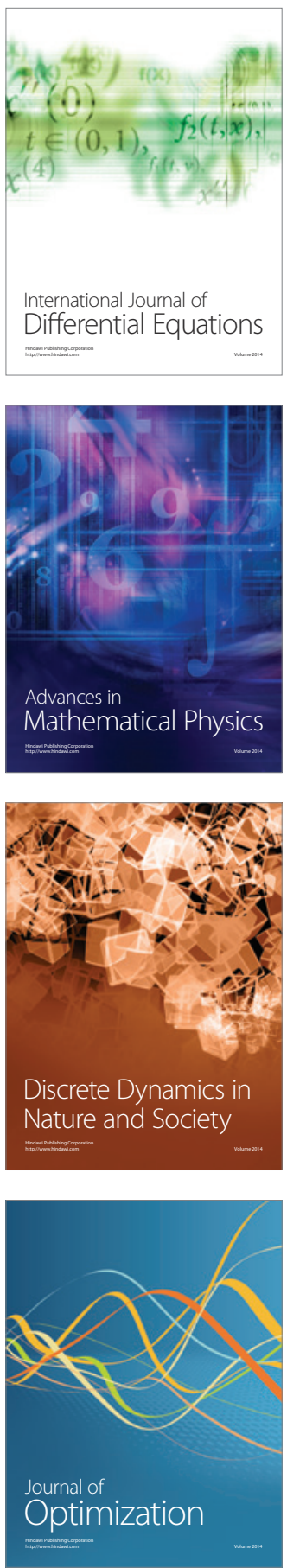